\title{
PROTOCOL CONVERTER (UART, I2C, MANCHESTER PROTOCOLS TO USB)
}

\author{
Shilpa Kanse ${ }^{1}$, Bhalerao Mayuri ${ }^{2}$, Bhosale Abhilasha ${ }^{3}$, Kardile Nilam ${ }^{4}$ \\ ${ }^{1}$ Professor, electronics telecommunication, RSCOE, tathwade, pune-33, Maharashtra, India \\ ${ }^{2}$ Student, electronics telecommunication, RSCOE, tathwade, pune-33, Maharashtra, India \\ ${ }^{3}$ Student, electronics telecommunication, RSCOE, tathwade, pune-33, Maharashtra, India \\ ${ }^{4}$ Student, electronics telecommunication, RSCOE, tathwade, pune-33, Maharashtra, India
}

\begin{abstract}
Abstract now a day's many industries are using different types of protocols to show data on computer. For this purpose different modules are used which increases the hardware complexity and cost. This project (PROTOCOL CONVERTER) is helpful to overcome these problem different types of protocols such as Manchester, UART and I2Cconverted to the USB format which is compatible to the laptops which is the major application .By using different components such as PIC microcontroller 18F452, LCD, Personal computer, Max 232, DB9 connector.
\end{abstract}

Keywords::USB (universal serial bus)1, UART(universal asynchronous receiver/transmitter2), I2C (Inter-Integrated Circuit3), Manchester4.

\section{INTRODUCTION}

In many automation industries different types of protocols are used to show data on computer. For this purpose different modules are used which increases the hardware complexity.

To reduce complexity the data in the different forms can converted into USB (universal serial bus), which is compatible to laptops.

Laptops doesn't have serial port, different type of data formats is not applicable so for compatibility to the laptop USB is provided, because it has different data rates ( high speed 480Mbps, medium speed $12 \mathrm{Mbps}$ and Low speed is $1.5 \mathrm{Mbps})$.

This module can be divided into two stages, generator and converter circuitry. First is the generator circuitry which produces different data in the different format like I2C, Manchester, and UART. Second part is protocol converter circuitry which converts this different data formats into a USB formats.

\section{PROTOCOL DESCRIPTION}

\subsection{USB}

Universal Serial Bus (USB) is an industry standard that defines the cables, connectors and communications protocols used in a bus for connection, communication, and power supply between computers and electronic devices.USB has Effectively replaced a variety of earlier interfaces, such as serial and parallel ports, as well as separate chargers for portable devices[3]. USB input output formats are shown in figure2.1.1.

\begin{tabular}{|l|l|l|l|l|l|}
\hline FIELD & SYNC & PID & DATA & CRC & EOP \\
\hline
\end{tabular}

Fig.2.1.1 USB Frame format

\subsection{I2C (Inter-Integrated Circuit):}

[3]Today, at the low end of the communication protocols, we find I2C (for 'Inter-Integrated Circuit', protocol). This protocol is well-suited for communications between integrated circuits, for slow communication with on-board peripherals.

I2C bus input output formats are shown in figure2.2.2.

\begin{tabular}{|l|l|l|l|}
\hline START & DATA & ACK & STOP \\
\hline \multicolumn{4}{|c|}{ Fig.2.2.1 I2C bus Frame format }
\end{tabular}

[1]Virtually any number of slaves and any number of masters can be connected onto these 2 signal lines and communicate between each other using a protocol that defines: - 7-bits slave addresses: each device connected to the bus has got such a unique address;

- Data divided into 8-bit bytes

- A few control bits for controlling the communication start, end, direction and for an acknowledgment mechanism.

The data rate has to be chosen between $100 \mathrm{kbps}, 400 \mathrm{kbps}$ and 3.4 Mbps, respectively called standard mode, fast mode and high speed mode.

\subsection{Manchester}

In telecommunication and data storage, Manchester coding (also known as Phase Encoding, or PE) is a line code in which the encoding of each data bit has at least one transition and occupies the same time. Manchester input output waveforms are shown in figure2.3.1. 

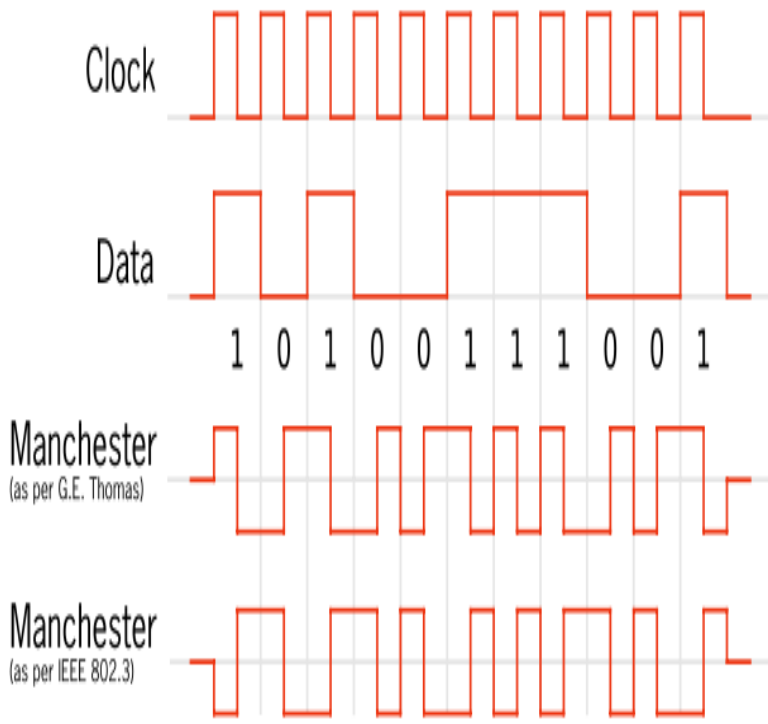

Fig.2.3.1 Manchester Input output waveform

\subsection{UART (Universal Asynchronous}

\section{Receiver/Transmitter)}

A universal asynchronous receiver/transmitter, abbreviated UART, is a piece of computer hardware that translates data between parallel and serial forms. UARTs are commonly used in conjunction with communication standards such as EIA, RS-232, RS-422 or RS-485. A UART is usually an individual (or part of an) integrated circuit used for serial communications over a computer or peripheral device serial port. UARTs are now commonly included in microcontrollers.USB input output formats are shown in figure2.4.1.

\begin{tabular}{|l|l|l|}
\hline START & DATA & STOP \\
\hline
\end{tabular}

Fig.2.4.1 UART Frame format

UART frame format consist of,

One start bit and one stop bit.

-parity is odd.

- 8-bit data (message bit)

\section{THE REASON FOR USING PIC CONTROLLER}

In this paper we are using PIC controller 18F458 to implement protocol converter

PIC controller has inbuilt 32 Kbytes of flash program memory. It has SRAM 1536 bytes and EEPROM 256 bytes data memory.

PIC controller has 10 bit A/D converter (8 input channels).

It has programmable brownout reset and low voltage detect.

[5]

\section{MAX 232}

[5]MAX 232 is an IC, which converts signals 4 from an RS232 serial port to suitable signals. The MAX232 is a dual receiver/driver. The drivers provide a maximum voltage 0 to $+5 \mathrm{~V}$.

\section{BLOCK DIAGRAM}

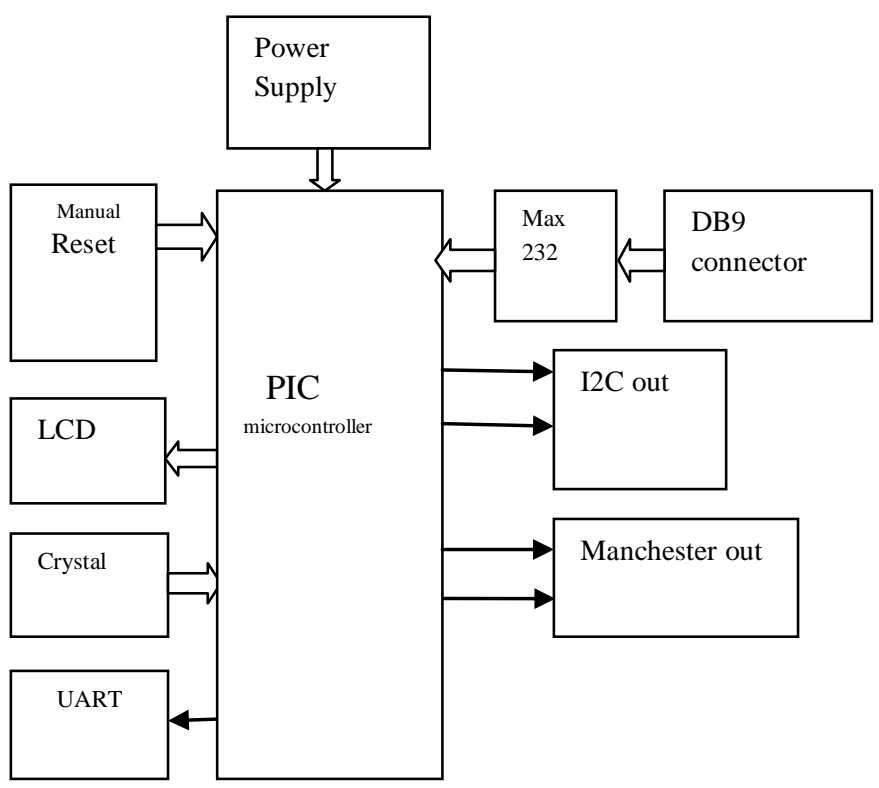

Fig 5.1 Block Diagram of generator

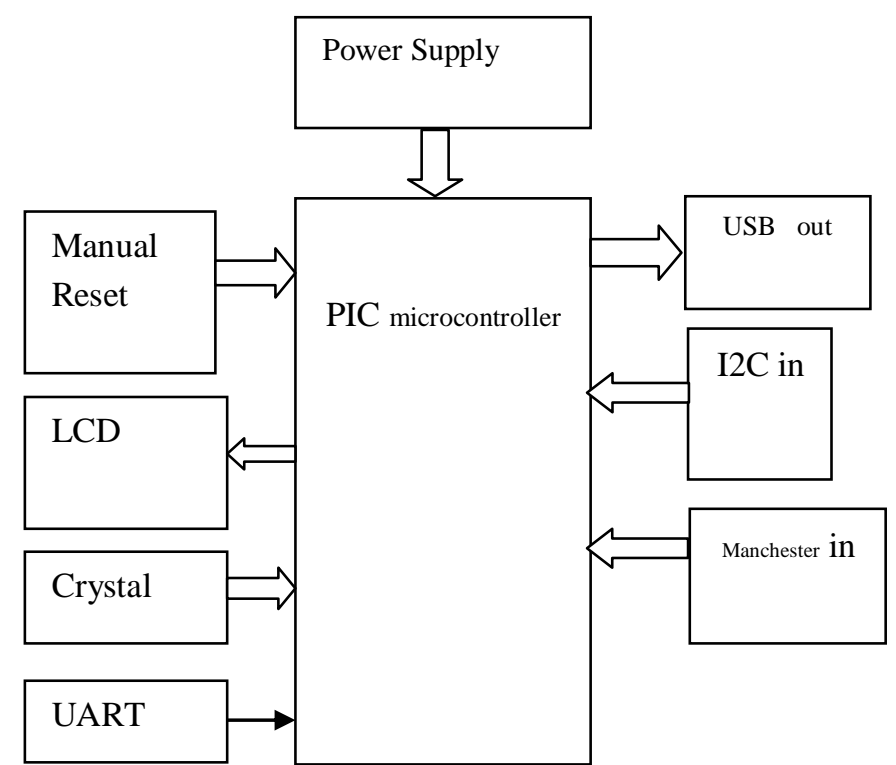

Fig 5.2 Block Diagram of converter 


\section{SYSTEM ARCHITETURE}

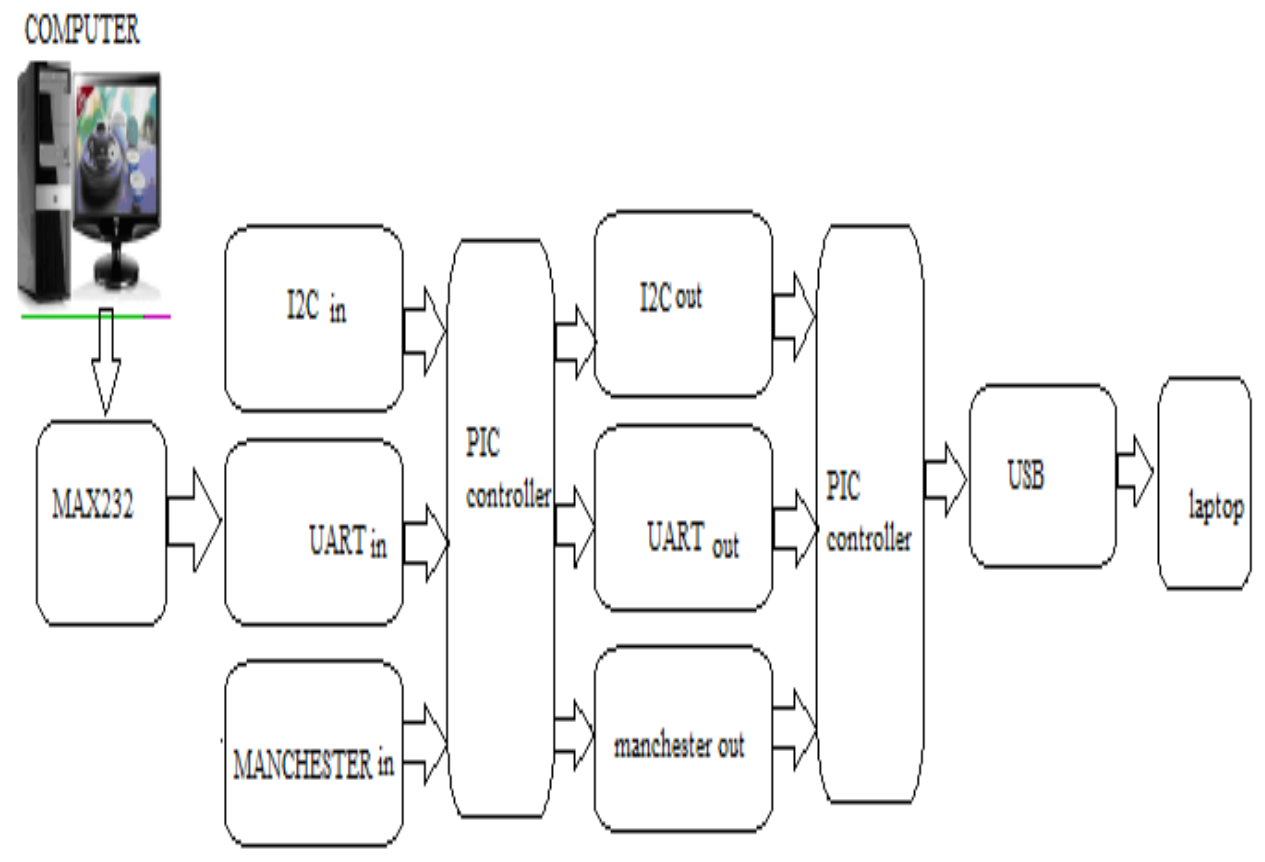

Fig 6.1 system architecture

In this system two PIC controllers are used. Selected PIC controller has inbuilt 32 Kbytes of flash program memory. This module can be divided into two stages, generator and converter circuitry. First is the generator circuitry which produces different data in the different format like I2C, Manchester, and UART. Second part is protocol converter circuitry which converts this different data formats into a USB format. The data is taken from computer and send to PIC controller of generator circuitry and then required data format is send to another controller of converter circuitry. This system is compatible to laptops. Laptop doesn't have a serial port. This system is also useful in an automobile industry.

\section{CONCLUSION}

This study proposed the design of a low cost protocol converter because of reduced hardware complexity. This is capable of receiving different data formats (Manchester, UART, and I2C) and converts data into USB format for notebook computer.

\section{REFERENCES}

[1]. Design and implementation of CAN-USB Converter Based on ARM7 Serial Protocol API, By Wei-Chih Hsu, Shih-Tsung Liu, In 2012.

[2]. www.ieee xplorer.org

[3]. I2C-USB Bridge Guide, by Petro Kobluk, Valeriy

Kyrynyuk, September 13, 2006.

[4]. 8051 Microcontroller, byMazidi.

[5]. www.datasheets.com 